\title{
Gravimetrical, theoretical, and surface morphological investigations of corrosion inhibition effect of 4-(benzoimidazole-2-yl) pyridine on mild steel in hydrochloric acid
}

\author{
Resen A.M. ${ }^{1}$, Hanoon M. ${ }^{1}$, Salim R.D. ${ }^{2}$, Al-Amiery A.A. ${ }^{3}$, Shaker L.M. ${ }^{3}$, Kadhum A.A.H. ${ }^{4}$ \\ ${ }^{1}$ Production Engineering and Metallurgy, University of Technology, Baghdad, Iraq \\ ${ }^{2}$ Electrical Engineering Technical College, Middle Technical University, Iraq \\ ${ }^{3}$ Energy and Renewable Energies Technology Center, University of Technology, Baghdad, Iraq \\ ${ }^{4}$ University of Al-Ameed, Karbala, Iraq \\ E-mail: dr.ahmed1975@gmail.com
}

The corrosion inhibition efficiency of the novel pyridine namely, 4-(Benzoimidazole-2-yl)pyridine has been studied for mild steel in a $1 \mathrm{M}$ hydrochloric acid environment by utilizing gravimetrical techniques. The synthesized inhibitor exhibits a significant inhibitive efficiency of $93.8 \%$ at $0.005 \mathrm{M}$. The adsorption isotherm of the investigated inhibitor on mild steel surface obeys the Langmuir isotherm. Surface morphology investigated by utilizing scanning electron microscopy (SEM) demonstrates a smooth metal surface with the addition of 4-(Benzoimidazole-2-yl)pyridine in a hydrochloric acid environment. Quantum chemical calculations using density functional theory (DFT) have been used to investigate the molecular structure and behavior of 4-(Benzoimidazole-2-yl) pyridine as a corrosion inhibitor. Different parameters have been calculated using DFT, such as energies of highest occupied molecular orbital and lowest occupied molecular orbital (EHOMO and ELUMO), energy gap $(\Delta E)$, and dipole moment $(\mu)$. These parameters were important to elucidate the behavior of the investigated molecule as a corrosion inhibitor in acidic solution and also suggest the mechanism of inhibition.

\section{INTRODUCTION}

The corrosion of alloys is major, academic, and manufacturing concern which was received significant interest. Mild steel is quiet, used as a structural alloy in considerable oil and gas manufacture because of its important mechanical characteristics and economically useful [1-3]. Generally, the corrosive medium is utilized in industries, such as pickling, cleaning, production, and acidification [4-7]. Corrosion issues manifest as a consequence of the reaction of the acidic environments and metal surface, particularly, through the process of pickling in which the metal surface is brought in contact with the acids that have high concentrations. For these reasons, the corrosion of mild steel produces economic damages [8-11]. The employ of corrosion inhibitors is the most significant technique for defense versus corrosion, particularly in corrosive solutions. In general, the organic compounds which have heteroatoms such as phosphorous, sulfur, oxygen, and/or nitrogen atoms were demonstrated to be efficient corrosion inhibitors of alloys such as mild steel in corrosive solutions [12-14]. These heterocyclic molecules have the abilities to be adsorbed on the surface of alloys and block the efficient site on the alloys surface, and control the corrosion. pyridine analogs have continued to be the topics of extended studies in applied science due to their wide applications as anticancer, antibacterial [15-17], and considerable usages such as inhibition for corrosion of mild steel. Some of the pyridine analogs were exhibited remarkable abilities to block corrosion of alloys in corrosive media $[18,19]$. The literature survey shows that, despite the significant activity of pyridine derivatives to react vigorously with the surface of the alloys, small interesting was paid to apply of pyridine derivatives as inhibitors for corrosion in corrosive solutions. The inhibitive performance of pyridine derivatives is imputed to the chemical structures of these molecules. The unshared electron pairs of the heteroatom and/or pi-bonds are the significant feature which evaluated the adsorption of such molecules on the surface of metals. The main theme of the present investigation is to study the inhibitive conduct of 4-(Benzoimidazole-2-yl) pyridine on the surface of mild steel in $1.0 \mathrm{~N} \mathrm{HCl}$ solution utilizing weight loss methods and scanning electron microscopic technique. The adsorption process is performed and the thermodynamical parameters have been evaluated. Additional confirmation of the inhibitive performance of 4-(Benzoimidazole-2-yl)pyridine is conducted by theoretical calculations. 


\section{EXPERIMENTAL SECTION}

\section{Materials and solutions}

The mild steel specimen composition in weight. \%: C, 0.21; Mn; 0.05; Si 0.38; S 0.05; Al 0.01; P 0.09; balance Fe. The mild steel specimen has been cut to the dimensions $25 \times 20 \times 0.25 \mathrm{~mm}$ for gravimetric techniques. The emery papers were used to abraded the mild steel specimen surface. Washed double distilled water and acetone. The used solution was hydrochloric acid (1 M) and was prepared through dilution of standard hydrochloric acid $37 \%$ with distilled water.

\section{Weight loss techniques}

Weight loss methods have been conducted with mild steel specimens through the exposure of the specimen to the $1 \mathrm{M}$ hydrochloric acid solution without and with the addition of 4-(Benzoimidazole-2-yl)pyridine at various concentrations $(0.001,0.002,0.003,0.004$, and $0.005 \mathrm{M})$. After a period $(1,5,10,24$, and $48 \mathrm{~h})$ the mild steel specimen has been taken out washed with double distilled water and acetone than dried by the oven and the weight was measured accurately [20-22].

Commonly used unit of corrosion rate expression measuring Weight Loss per Unit Area per Unit Time and referred to as milligrams per square decimeter per day or "mdd". A milligram is one-thousandth of a gram; a decimeter is 10 centimeters. If mdd is multiplied by $0.03652 / \rho$ ( $\rho=$ density in grams per cubic centimeter or $\mathrm{g} \mathrm{cm}^{-3}$ ) it is the same as millimeters per year or "mmpy" the corrosion rate $=K W / \rho A t$, where $K$ is a constant, its magnitude depends on the system of units used $K=87.6$ for $\mathrm{C}_{\mathrm{R}}$ in $\mathrm{mm} / \mathrm{yr}$ (or mmpy) and unit for the other parameters are the same for the first case except that $A$ is given in square centimeters.

The rate of corrosion rate was calculated according to equation 1 :

$$
\text { Corrosion rate }(\mathrm{mmpy})=\frac{(87.6 \times W)}{\rho A t}
$$

where $W$ is the weight loss of mild steel tested coupon, $A$ is the surface area of mild steel tested coupon, $t$ is the exposure time, and $\rho$ is the density (in $\mathrm{g} / \mathrm{cm}^{3}$ ) of mild steel tested coupon.

\section{Quantum chemical calculations}

The density-functional theory (DFT) method was conducted on the investigated 4-(Benzoimidazole-2-yl) pyridine with the correlation Lee-Yang-Parr functional (B3LYP). The basis set was $6-31+G(d, p)$ for the calculations which were performed with the Gaussian software. The optimized geometrical structure of the 4-(Benzoimidazole-2-yl)pyridine molecule was confirmed. The energies of frontier molecular orbital (FMO), which were the energy of the highest occupied molecular orbital (HOMO) and energy of the lowest unoccupied molecular orbital (LUMO) were evaluated [23,24]. The quantum chemical parameters namely energy gap $(\Delta E)$, electronegativity $(\chi)$, and the fraction of electrons transferred $(\Delta N)$ were calculated according to the equations (2-4).

$$
\begin{gathered}
\Delta E=E_{\mathrm{LUMO}}-E_{\mathrm{HOMO}} \\
\chi=-0.5\left(E_{\mathrm{LUMO}}+E_{\mathrm{HOMO}}\right) \\
\Delta N=\frac{\chi_{\mathrm{Fe}}-\chi_{\mathrm{inh}}}{2\left(\eta_{\mathrm{Fe}}+\eta_{\mathrm{inh}}\right)}
\end{gathered}
$$

where $\chi_{\mathrm{Fe}}$ is the iron electronegativity and $\eta_{\text {inh }}$ is the inhibitor hardness.

The $\chi_{\mathrm{Fe}}$ and $\eta_{\mathrm{Fe}}$ values were $7 \mathrm{eV} / \mathrm{mol}$ and $0 \mathrm{eV} / \mathrm{mol}$ respectively for iron.

\section{Scanning electron microscopy (SEM)}

Surface analyses were performed to investigate the variations of the surface morphology of the mild steel coupon in the absence and in presence of tested inhibitor. After $5 \mathrm{~h}$ of exposure in $1 \mathrm{M}$ acidic solution at $30{ }^{\circ} \mathrm{C}$, the coupons were washed and dried.

\section{RESULTS AND DISCUSSION}

\section{Weight loss methods - Effect of inhibitor concentration and immersion time}

The corrosion rate $\left(C_{R}\right)$ and inhibition efficiency (IE\%) which were evaluated from weight loss results are plotted in Figure 1. These results exhibit that, in addition to various synthesized inhibitor concentration,

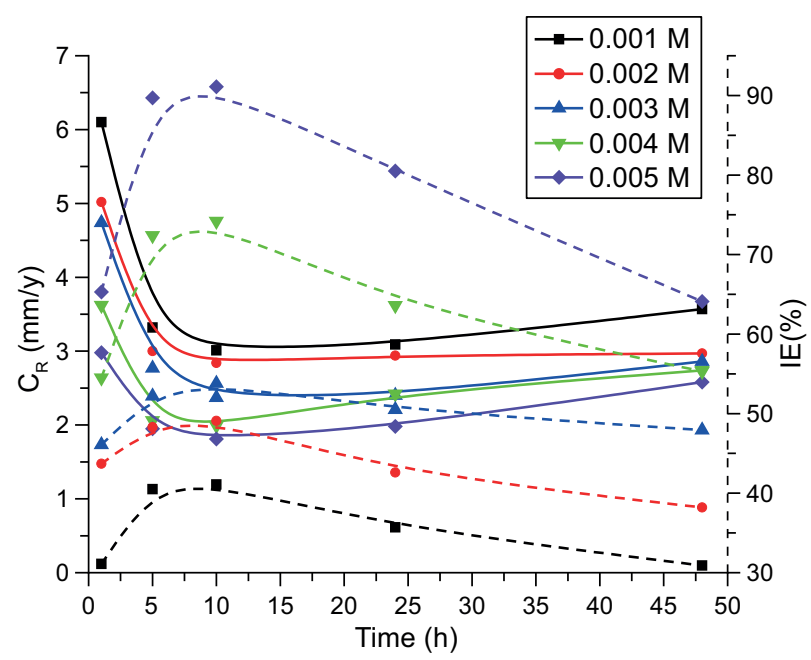

Fig. 1. Effect of various concentrations of 4-(Benzoimidazole-2-yl)pyridine on corrosion rate and inhibition efficiency at different immersion time for mild steel in $1 \mathrm{M}$ hydrochloric acid solution 
the corrosion rate $\left(C_{R}\right)$ decreases, and inhibition efficiency (IE\%) increases. This refers that the inhibition efficiency of the investigated inhibitor is dependent on the synthesized inhibitor concentration and this is obviously, shown in the plot of corrosion rate, inhibition efficiency, time, and concentrations demonstrated in Figure 1. The inhibitive performance of the synthesized pyridine namely, 4-(Benzoimidazole-2-yl)pyridine was studied for mild steel in $1 \mathrm{M}$ hydrochloric acid solution with different immersion time (1, 5, 10, 24, and $48 \mathrm{~h})$ and various inhibitor concentrations (0.001-0.005 M). Time has a significant impact on the efficiency of the used inhibitor, as increasing the immersion time leads to increasing the inhibition efficiency of the studied inhibitor, and this is logical, since the increase in immersion time works to increase the number of adsorbed molecules on mild steel surface, and this is evident in Figure 1. Increasing the exposure time up to 24 hours leads to a decrease in the inhibition efficiency and this can be explained based on several explanations including the dissolution of the inhibitor molecules used with the increase in immersion time. The gravimetrical measurements are credible methods to optimize the concentration effect of the studied inhibitor on inhibitive performance [25-29].

The IE (\%) was evaluated according to equation (5).

$$
\operatorname{IE}(\%)=\frac{\mathrm{C}_{\mathrm{R}}-\mathrm{C}_{\mathrm{Ri}}}{\mathrm{C}_{\mathrm{R}}} \times 100
$$

where $C_{R}$ is the corrosion rate (mmpy) without the addition of the synthesized inhibitor and $\mathrm{C}_{\mathrm{Ri}}$ is the corrosion rate (mmpy) with the addition of the synthesized inhibitor.

The investigated synthesized inhibitor controls or impedance the corrosion of the mild steel surface through the surface coverage $(\theta)$ which increases the inhibitive performance, and the surface coverage can evaluate $[30,31]$ according to the equation (6).

$$
\theta=\frac{I E}{100}
$$

The increase in 4-(Benzoimidazole-2-yl)pyridine concentration proceed to an increase in the adsorption extent and surface coverage because of the considerable number of 4-(Benzoimidazole-2-yl)pyridine molecules and therefore an increase in inhibitive performance. The significant inhibition efficiency of the examined inhibitor is attributed to the presence of nitrogen atoms and aromatic rings in the structure of the studied inhibitor.

The current anticorrosive compound is the product of a combination of two heterocyclic compounds, both of which are included in the synthesis of some pharmaceutical drugs, which are pyridine and benzoimidazole, thus the resulting compound (our synthesized inhibitor) is an environmentally friendly inhibitor. On the other hand, the newly synthesized corrosion inhibitor is produced from a one-step reaction of inexpensive raw materials, therefore it can be produced industrially easily and at a low cost. It is well documented in the literature that organic inhibitors are more effective and cheaper compared to inorganic. Recently, a lot of research has been devoted to producing non-toxic green inhibitors. In this study, a mild steel anti-corrosion inhibitor was synthesized and compared with different organic inhibitors regarding their inhibition efficiency, which is evaluated from weight loss techniques, in a medium of hydrochloric acid. As noted from Table 1, the studied inhibitor 4-(Benzoimidazole-2-yl)pyridine has high inhibition efficiency compared to the corrosion inhibitors as well as the ability to manufacture easily and in one step. Although some of the synthesized and corrosion inhibitor in Table 1, have a relatively higher inhibition efficiency compared to the investigated inhibitor, also they are either toxic or environmentally unfriendly or expensive to synthesize, with reactions that require more than one step and require expensive solvents.

Tab. 1. Some of the published corrosion inhibitor compared to the investigated inhibitor

\begin{tabular}{|c|l|c|c|}
\hline No. & Inhibitor & IE\% & Reference \\
\hline 1 & 4-(Benzoimidazole-2-yl)pyridine & 91.16 & This study \\
\hline 2 & 4-Dimethylamino-benzylidene)-[1,3,4]thiadiazol-2-yl-amine & 91 & 2 \\
\hline 3 & 4-hydroxybenzylideneaminomethyl-5-ethyl-1,3,4-thiadiazol & 90 & 3 \\
\hline 4 & 2-Methyl-4H-benzo[d][1,3]oxazin-4-one & 65 & 7 \\
\hline 5 & 3-amino-2-methylquinazolin-4(3H)-one & 89 & 7 \\
\hline 6 & 3-((5-(3,5-dinitrophenyl)-1,3,4-thiadiazol-2-yl)imino)indolin-2-one & 90.7 & 8 \\
\hline 7 & 3-nitro-5-(2-amino-1,3,4-thiadiazolyl)nitrobenzene & 92.3 & 9 \\
\hline 8 & 3-((4-(dimethylamino)benzylidene)amino)-2-methylquinazolin-4(3H)-one & 92 & 25 \\
\hline 9 & 4-((5,5-dimethyl-3-oxocyclohexenyl)amino)benzenesulfonamide & 93 & 27 \\
\hline 10 & 2-((2-hydroxy-5-methoxybenzylidene)amino)pyridine & 90 & 28 \\
\hline
\end{tabular}




\section{Weight loss methods}

- the effect of temperature

The inhibitive efficiency increases with the increasing 4-(Benzoimidazole-2-yl)pyridine concentration. From these findings, we realize that the required optimum inhibitor concentration to conduct the highest inhibition performance was $0.005 \mathrm{M}$. The increase in the inhibitive effectiveness supposedly imputed to the adsorption of 4-(Benzoimidazole-2-yl)pyridine molecules on the mild steel surface to form a protected layer on the tested. The increase in the inhibitive performance, probably imputed to the slow replacement of $\mathrm{H}_{2} \mathrm{O}$ molecules by the adsorption of the 4-(Benzoimidazole2-yl)pyridine molecules on the mild steel surface, reducing the degradation reaction range. The mild steel corrosion inhibition by 4-(Benzoimidazole-2-yl)pyridine as a synthesized corrosion inhibitor may be demonstrated in terms of adsorption on the mild steel surface. 4-(Benzoimidazole-2-yl)pyridine can be adsorbed on the mild steel through the reaction between unshared nitrogen atoms electrons of nitrogen atoms of the 4-(Benzoimidazole-2-yl)pyridine molecules and the d-orbital of iron atoms of the mild steel surface. To study the inhibition mechanism, gravimetric techniques were taken at different temperatures without and with the addition of various concentrations of 4-(Benzoimidazole-2-yl)pyridine at various exposure time. In the investi-gated temperature extent $\left(30-60{ }^{\circ} \mathrm{C}\right)$ the rate of corrosion increases with increasing solution temperature and the inhibition efficiency of 4-(Benzoimidazole-2-yl)pyridine is decreased. 4-(Benzoimidazole-2-yl)pyridine acts as an excellent corrosion inhibitor of mild steel in $1 \mathrm{M}$ hydrochloric acid environment and the highest inhibitive efficiency is $91 \%$ at $0.005 \mathrm{M}$ for 5 hours as exposure time $30{ }^{\circ} \mathrm{C}$. The value of inhibition efficiency increase with the inhibitor concentration, but decreases with increasing immersion time and/or solution temperature of mild steel corrosion $1 \mathrm{M}$ hydrochloric acid environment. Time is a significant parameter when describing the corrosion inhibitor. To evaluate the tested inhibitor stability, the exposure period investigated was achieved. The exposure times were different from 1 to $24 \mathrm{~h}$. The highest inhibition efficiency was attained at the exposure time which was $5 \mathrm{~h}$. This was imputed to the immediate adsorption of the inhibitor molecules on to surface of metal due to the highest number of ready efficient inhibitor molecules to block metal surface from corrosion. After $10 \mathrm{~h}$. of exposure time the inhibition efficiency was observed to diminish, it was more diminished after the higher exposure time that was $24 \mathrm{~h}$. The diminished inhibition efficiency was imputed to desorption of the inhibitor molecules from the mild steel surface. Furthermore, the corrosion rate increased with a long time of exposure, which was also due to desorbed of studied inhibitor molecules from the surface of mild steel, a coupon mild steel was immersion in hydrochloric acid solution thereby increasing mild steel-acidic environment contact that produces farther mild steel being dissolved. Furthermore, the increase in corrosion rate after long exposure time was imputed to a less concentration of studied inhibitor in a corrosive environment to control or impedance mild steel dissolution; it is obvious that after inhibitor molecules were desorbed from the surface of mild steel they become inefficient so, these molecules did not invalid in the inhibitive approach

The corrosion rate and inhibition efficiency are affected by the temperature of the solution. As in Fig. 2, it is obvious that the corrosion rate increases, and inhibition efficiency decrease for 4-(Benzoimidazole-2-yl) pyridine as a corrosion inhibitor increase in temperature. This imputed to desorption for the inhibitor molecules from the surface of mild steel at higher temperatures [32].

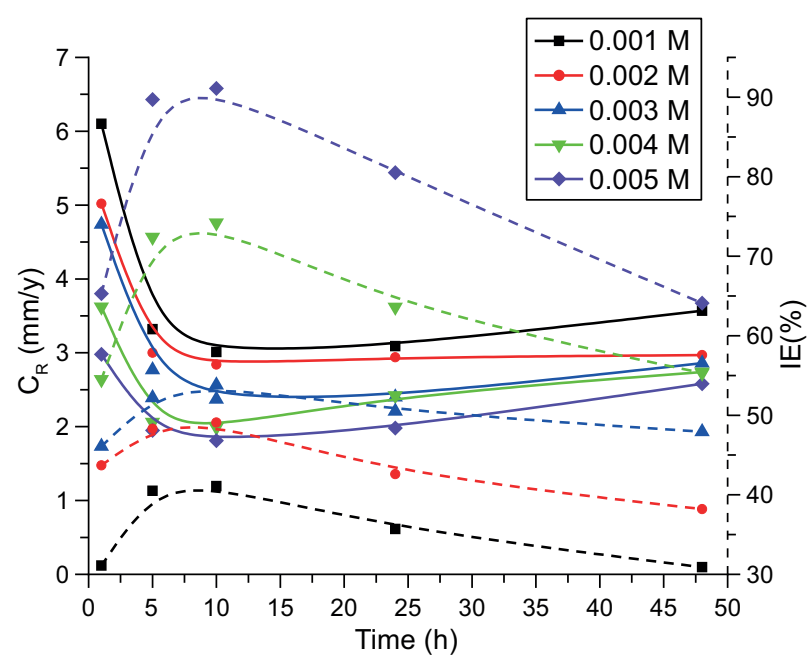

Fig. 2. Effect of various concentrations of 4-(Benzoimidazole-2-yl)pyridine on corrosion rate and inhibition efficiency at different immersion time for mild steel in $1 \mathrm{M}$ hydrochloric acid solution

\section{Adsorption isotherm}

To understand the interaction model between the surface of tested coupon and inhibitor molecules the adsorption isotherms are utilized. The inhibitor molecules which be adsorbed on the surface of mild steel never reach the actual equilibrium, however, resort to a steady state of adsorption. The presence of inhibitor molecules leads to decreases in the corrosion rate and the adsorption process tends to achieve a semi-balanced state. The nature of the semi-equilibrium adsorption of the inhibitor molecules can be verified utilizing compatible isotherm. Experimental findings were exposed to different adsorption isotherms, and the feature is demonstrated in Figure 3. 


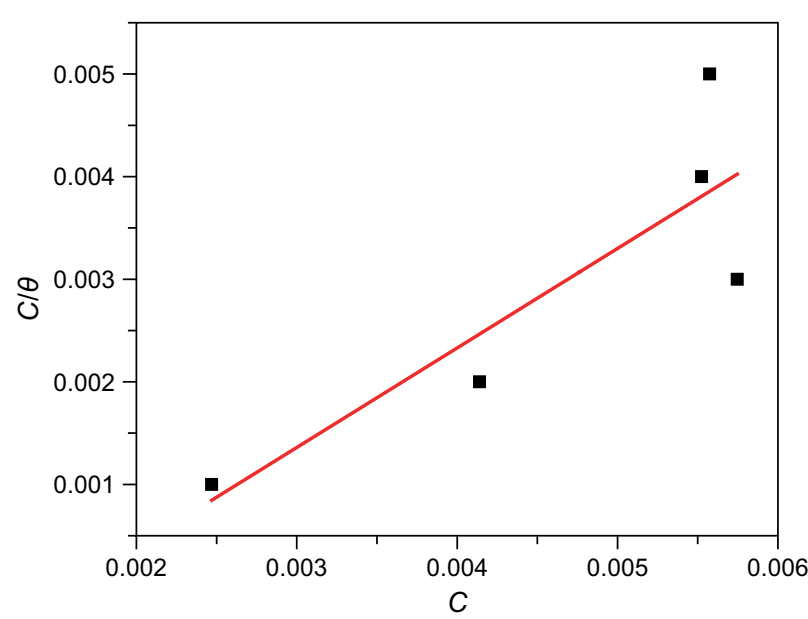

Fig. 3. Langmuir adsorption isotherm plot for mild steel in the presence of 4-(Benzoimidazole-2-yl)pyridine

To find the appropriate adsorption isotherm, three models namely "Temkin, Freundlich, and Langmuir" were applied according to equations (7-9). Figure 3, represents the Langmuir isotherm which was the suitable one.

$$
\begin{gathered}
\text { Temkin isotherm; } \exp ^{(f \theta)}=K_{\mathrm{ads}} C \\
\text { Freundlich isotherm; } \theta=K_{\mathrm{ads}} C \\
\text { Langmuir isotherm; } \frac{C}{\theta}=\frac{1}{K_{\mathrm{ads}}}+C
\end{gathered}
$$

where $K_{\text {ads }}$ is the equilibrium constant and $C$ is the concentration of studied inhibitor.

Equation (9) basically briefs the dependency of the concentration of inhibitor $(C)$ with the surface coverage $(\theta)$ of the inhibitor on the metal surface. The plot of $C / \theta$ vs $C$ (Fig. 3 ) obtained at $30{ }^{\circ} \mathrm{C}$ gives a straight line, and hence, adsorption of inhibitor obey Langmuir adsorption isotherm more accurately as compared to Temkin and Freundlich adsorption isotherms. The equilibrium constant $\left(K_{\text {ads }}\right)$ indicates the relationship between the adsorbate and adsorbent. The higher $K_{\text {ads }}$ value signifies greater adsorption and hence significant inhibition efficiency $[33,34] . K_{\text {ads }}$ can be achieved utilizing the straight-line intercept which is $23.9\left(104 \mathrm{M}^{-1}\right)$. The correlation between equilibrium constant $\left(K_{\text {ads }}\right)$ and free energy $\left(\Delta G_{\text {ads }}\right)$ can be evaluated according to equation (10).

$$
\Delta G_{\mathrm{ads}}=-R T \ln \left(55.5 K_{\mathrm{ads}}\right)
$$

where 55.5 value is the molar water concentration whereas the $R$ and $T$ are the gas constant and absolute temperature respectively.

Gibbs free energy $\left(\Delta G_{\text {ads }}{ }^{\circ}\right)$ with adsorption negative value signifies process spontaneity and hence the inhibitor molecules are efficiently adsorbed on the mild steel surface [35]. The physisorption (electrostatic) interaction of the inhibitor molecule and the mild steel surface is characterized by $\Delta G_{\mathrm{ads}}{ }^{\circ} \leq-20 \mathrm{~kJ} \mathrm{~mol}^{-1}$.
An adsorption free energy with a highly negative value $\left(>40 \mathrm{~kJ} \mathrm{~mol}^{-1}\right.$ ) signifies a co-ordinate bond type, that is, chemisorption [36]. In this case, $\Delta G_{\text {ads }}{ }^{\circ}$ value is $41.73 \mathrm{~kJ} \mathrm{~mol}^{-1}$. This suggests that the mechanism involves chemisorption and physisorption which is a mixed interaction mode.

\section{Quantum chemical calculations}

The frontier molecular orbital was conducted to investigate the reactivity of the inhibitor molecule and its different interactions with the mild steel surface. According to frontier molecular orbital theory (FMO), the chemical reactivity is the interaction between the highest occupied molecular orbital (HOMO) and lowest unoccupied molecular orbital (LUMO) levels of the reacting species. The optimized geometry, highest occupied molecular orbital and lowest unoccupied molecular orbital of 4-(Benzoimidazole-2-yl)pyridine molecules are demonstrated in Figure 4, and the different quantum factors are shown [37]. It is obvious from Fig. 4, that the highest occupied molecular orbital density is generally delocalized over the pyridine ring and the lowest unoccupied molecular orbitalis over benzoimidazole and pyridine rings. The energy of the highest occupied molecular orbital value represents the ability of the investigated molecules to donate electrons and to form coordination bonds with the unoccupied d-orbital of iron atoms and so the highest value of the energy of the highest occupied molecular orbital denotes improved inhibitive performance. The energy of the lowest unoccupied molecular orbital is the capacity of accepting electrons of the molecule and hence the value low of the energy of the lowest unoccupied molecular orbital proposes an appropriate condition for accept electron through back-donation from iron. The 4-(Benzoimidazole-2-yl)pyridine molecules were found to have relatively high energy of the highest occupied molecular orbital and reasonably low value of the energy of the lowest unoccupied molecular orbital that gives it to have the capability to give electrons to the d-orbital of iron atoms as well as accept electrons under appropriate provisions that makes it an excellent corrosion inhibitor. The energy gap which was the difference between the energy of the highest occupied molecular orbital and energy of the lowest unoccupied molecular orbital is a measurement of inhibition efficiency and the ability of inhibitor molecules to inhibit the corrosion of corrosive solution. The low value of the energy gap indicates that the molecule has low kinetic stability with the nature of polarisable in addition to easy adsorption on mild steel surface [38]. The energy gap is the main key-factor in predicting chemical stability and reactivity of inhibitor molecules. As the energy gap value is reduced, the molecule is more active toward the inhibition process $[39,40]$. The energy gap with a 
high value refers to the less polar the molecule which signifies that the adsorption of this molecule on the mild steel surface is uneasy [41]. Hence, an excellent inhibitor must have an energy gap with low value. The molecular structure, $E_{\mathrm{HOMO}}$, and $E_{\mathrm{LUMO}}$ of the investigated molecule are demonstrated in Figure 4. The optimized molecular structure in Figure 4, demonstrated that the studied inhibitor molecule takes on a planar geometry. The inhibition efficiency of the studied inhibitor molecule may impute to a planar geometry of these molecules. The planar molecules are more and these molecules can coordinate with the metal surface and form a coordination bond with iron atoms at the metal surface and leading to enhanced inhibitive performance. The $E_{\text {номо }}$ value helps to evaluate the ability of a molecule to share electrons. A molecule with higher $E_{\text {Номо }}$ can easily share electrons to appropriate acceptor with, unoccupied orbital [42]. The $E_{\text {Номо }}$ of a molecule gives data about the functional groups of the inhibitor molecule which have unshared pairs of electrons. These electron pairs are coordinated with d-orbital of iron atoms. On the other hand, the $E_{\text {LUMo }}$ value represents the capability of a molecule to share electrons. The $E_{\text {LUMO }}$ with low value can accept electrons easily. [42]. The $\mathrm{L}_{\mathrm{UMO}}$ of a molecule suggests the functional groups of the inhibitor molecules which can accept electrons. $E_{\text {номо }}$ is an evaluation of a molecule tendency to donate unpaired of electrons to the unoccupied the d-orbital of the metal, so, the highest inhibition efficiency of a molecule comes with the higher $E_{\text {Номо }}$ of that molecule. The HOMO (-10.273) of 4-(Benzoimidazole-2-yl)pyridine is quite distributed over the pyridine ring in the molecule so, the except the benzoimidazole does not contribute to the HOMO.

The $\Delta N$ value which represents the electrons transferred fraction from the molecule of the tested inhibitor to the mild steel surface was 0.9013 , also confirm the tendency of the methodological inhibition efficiency. The utilize of the dipole moment $(\mu)$ is associated with the relative methodological inhibitive efficiency of organic molecules is generally characterized with opposed opinions. One of the opposed opinions supposes that $(\mu)$ with high value leads to a low inhibition efficiency with the clarification that the $(\mu)$ with low value will support inhibitor molecules accumulation on the mild steel surface. On the other hand, a different view demonstrates that $(\mu)$ with high value will increase the inhibition efficiency and this is because of the dipoledipole interactions between the tested inhibitor molecules and the surface of mild steel. The energy of the highest occupied molecular orbital $\left(E_{\mathrm{HOMO}}\right)$, electronegativity $(\chi)$, and a fraction of transferred electrons $(\Delta N)$ as quantum chemical parameters exhibit harmonic with the methodological findings and suggest the excellent inhibitive performance of the tested inhibitor molecules with an ability for donating electrons. This proposes that the mechanism of adsorption of the investigated molecules on the mild steel surface is generally through donating electrons from the nitrogen atoms and aromatic groups of the inhibitor molecules to the iron atoms of the mild steel surface.

\section{SEM micrographs}

The microstructure characterization of mild steel surface treated in $1 \mathrm{M}$ hydrochloric acid in the absence and presence of 4-(Benzoimidazole-2-yl)pyridine as a corrosion inhibitor is demonstrated in Figure 5. Figure $5 \mathrm{a}$ is the SEM micrograph of mild steel surface after immersion $5 \mathrm{~h}$ at $30{ }^{\circ} \mathrm{C}$, in corrosive solution in absence of 4-(Benzoimidazole-2-yl)pyridine which demonstrated cracks due to corrosion. Figure $5 \mathrm{~b}$ demonstrated the metallic surface with the addition of 4-(Benzoimidazole2 -yl)pyridine in the corrosive solution. From the SEM micrograph in Figure $5 \mathrm{~b}$, the metallic surface is smooth. This variation proves the adsorption of 4-(Benzoimidazole-2-yl)pyridine molecules on the mild steel and a form a protective film.

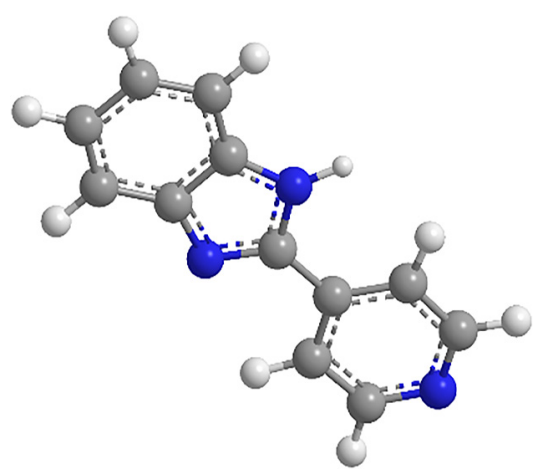

a)

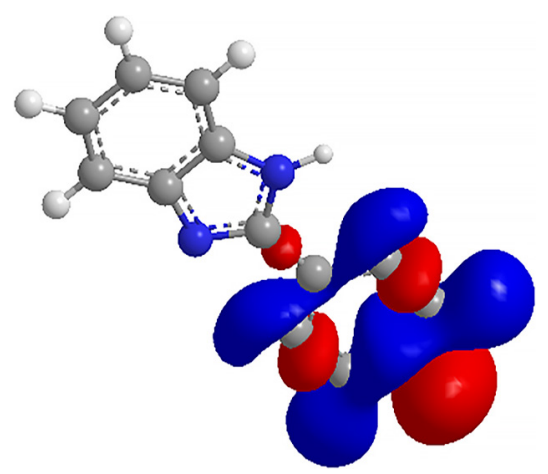

b)

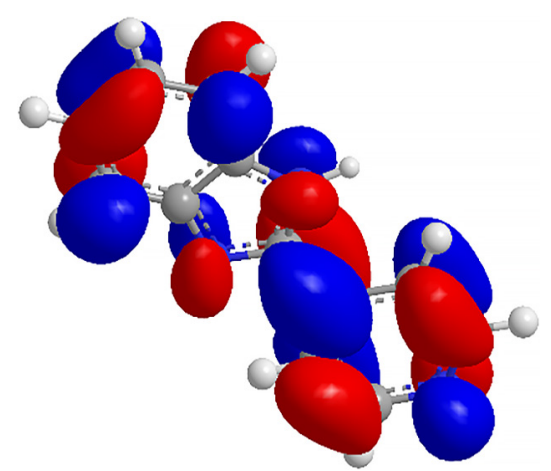

c)

Fig. 4. Optimized structure, HOMO, and LUMO surfaces of the 4-(Benzoimidazole-2-yl)pyridine 


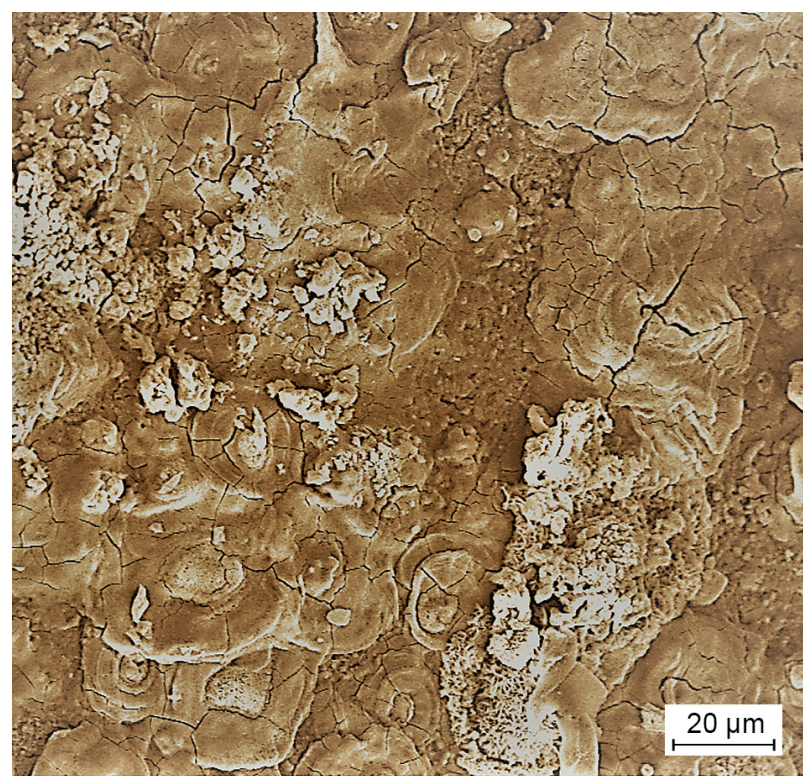

a)

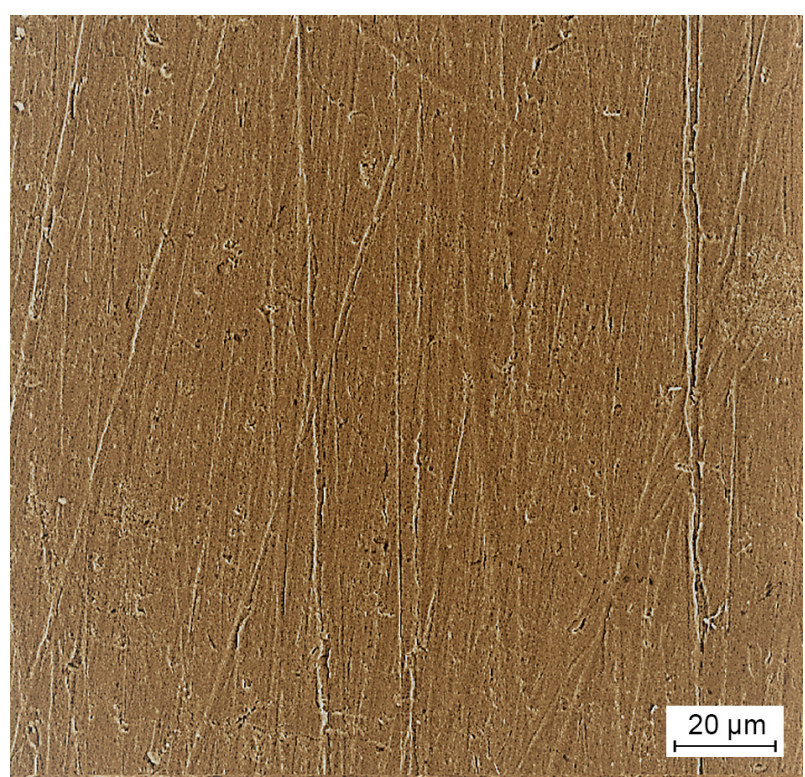

b)

Fig. 5. SEM micrographs of mild steel surface after $5 \mathrm{~h}$ of exposure in $1 \mathrm{M}$ hydrochloric acid solution in absence of 4-(Benzoimidazole-2-yl)pyridine (a) and in presence of -(Benzoimidazole-2-yl)pyridine (b)

\section{CONCLUSIONS}

New pyridine derivative namely "4-(Benzoimidazole-2-yl)pyridine" have been synthesized and fully characterized by spectroscopically techniques and CHN elemental analysis. The synthesized pyridine was studied as a potent of corrosion inhibition on mild steel in $1 \mathrm{M}$ hydrochloric acid solution. The findings revealed that the 4-(Benzoimidazole-2-yl)pyridine control or impedance of the mild steel corrosion in $1 \mathrm{M}$ hydrochloric acid solution and the inhibitive performance increase with increasing concentration. The findings demonstrated that the highest inhibition efficiency of the studied inhibitor was $89.64 \%$ at $0.005 \mathrm{M}$ for the immersion time $5 \mathrm{~h}$ and $91.16 \%$ at $0.005 \mathrm{M}$ for the immersion time $10 \mathrm{~h}$. The SEM micrographs demonstrated that the 4-(Benzoimidazole-2-yl)pyridine molecules protect the mild steel surface and also block mild steel surface from having direct contact with a hydrochloric acid solution. The adsorption of the 4-(Benzoimidazole-2-yl)pyridine molecules on mild steel surface in hydrochloric acid solution followed Langmuir isotherm. The quantum chemical parameters such as the energy of the highest occupied molecular orbital $\left(E_{\mathrm{HOMO}}\right)$, electronegativity $(\chi)$, and a fraction of transferred electrons $(\Delta N)$ for the synthesized corrosion inhibitor is in harmonic with experimental results.

\section{Acknowledgments}

This study was supported by the University of Technology, Baghdad, Iraq.

\section{REFERENCES}

1. El-Naggar, M.; Almahli, H.; Ibrahim, H.; Eldehna, W.; Abdel-Aziz, H.: Pyridine-Ureas as Potential Anticancer Agents: Synthesis and In Vitro Biological Evaluation. Molecules 2018, 23, 1459; doi:10.3390/molecules23061459

2. Salman, T.A.; Zinad, D.S.; Jaber, S.H.; Al-Ghezi, M.; Mahal, A.; Takriff, M.S.; Al-Amiery, A.: Effect of 1,3,4 Thiadiazole Scafold on the Corrosion Inhibition of Mild Steel in Acidic Medium: An Experimental and Computational Study, $J$. BioTribo-Corrosion, 2019, 5, 1

3. Habeeb, H.J.; Luaibi, H.M.; Dakhil, R.M.; Kadhum, A.A.H.; Al-Amiery, A.; Gaaz, T.S.: Development of new corrosion inhibitor tested on mild steel supported by electrochemical study, Results Phys. 2018, 8, 1260

4. Al-Amiery, A.; Kadhim, A.; Mohamad, A.; Musa, A.; Li, C.: Electrochemical study on newly synthesized chlorocurcumin as an inhibitor for mild steel corrosion in hydrochloric acid, Materials, 2013, 6, 5466

5. Ansari, K. R.; Quraishi, M. A.; Singh, A.: Pyridine derivatives as corrosion inhibitors for $\mathrm{N} 80$ steel in $15 \% \mathrm{HCl}$ : Electrochemical, surface and quantum chemical studies. Measurement, 2015, 76, 136-147

6. Junaedi, S.; Kadhim, A.; Al-Amiery, A.; Mohamad A.; Takriff, M.: Synthesis and characterization of novel corrosion inhibitor derived from oleic acid: 2-Amino5-Oleyl1, 3,4-Thiadiazol (AOT), Int. J. Electrochem Sci. 2012, 7, 3543

7. Kadhim,A.;Al-Okbi,A.;Jamil,D.M.;Qussay,A.;Al-Amiery, A.; Gaaz, T.; Kadhum, A.; Mohamad, A.; Nassir, M.H.: Experimental and theoretical studies of benzoxazines corrosion inhibitors, Results Phys. 2017, 7, 4013

8. Al-Azawi, K.F.; Mohammed, I.M.; Al-Baghdadi, S.B.; Salman,T.A.;Issa,H.A.;Al-Amiery,A.; Gaaz,T.;Kadhum,A.: Experimental and quantum chemical simulations on the 
corrosion inhibition of mild steel by 3-((5-) (3,5-dinitrophenyl)-1,3,4-thiadiazol-2-yl)imino)indolin-2-one, Results Phys. 2018, 9, 278

9. Al-Baghdadi, S.B.; Hashim, F.G.; Salam, A.Q.; Abed, T.K.; Gaaz, T.S.; Al-Amiery, A.; Kadhum, A.A.H.; Reda, K.S.; Ahmed, W.K.: Synthesis and corrosion inhibition application of NATN on mild steel surface in acidic media complemented with DFT studies, Results Phys. 2018, 8, 1178

10. Al-Obaidy, A.H.M.J.; Kadhum, A.; Al-Baghdadi, S.B.; Al-Amiery, A.; Kadhum, A.A.H.; Mohamad, A.B.; Yousif, E.: Eco-friendly corrosion inhibitor: experimental studies on the corrosion inhibition performance of creatinine for mild steel in $\mathrm{HCl}$ complemented with quantum chemical calculations, Int. J. Electrochem Sci. 2015, 10, 3961

11. Al-Amiery, A.; Kadhum, A.A.H.; Mohamad, A.B.; Junaedi, S.: A Novel Hydrazinecarbothioamide as a Potential Corrosion Inhibitor for Mild Steel in $\mathrm{HCl}$, Materials, 2013, 6, 1420

12. Ahmed, M.H.O.; Al-Amiery, A.; Al-Majedy, Y.K.; Kadhum, A.A.H.; Mohamad, A.B.; Gaaz, T.S.: Synthesis and characterization of a novel organic corrosion inhibitor for mild steel in $1 \mathrm{M}$ hydrochloric acid, Results Phys. 2018, 8, 728

13. Salman, T.A.; Al-Amiery, A.A.; Shaker, L.M.; Kadhum, A.; Takriff, M.S.A.: Study on the inhibition of mild steel corrosion in hydrochloric acid environment by 4-methyl2-(pyridin-3-yl)thiazole-5-carbohydrazide. Int. J. Corros. Scale Inhib. 2019, 8, 1035

14. Salman, T.A.; Jawad, Q.A.; Hussain, M.A.; Al-Amiery, A.A.; Shaker, L.M.; Kadhum, A.; Takriff, M.S.: Novel ecofriendly corrosion inhibition of mild steel in strong acid environment: Adsorption studies and thermal effects. Int. J. Corros. Scale Inhib. 2019, 8, 1123

15. Al-Taweel, S.S.; Al-Janabi, K.W.S.; Luaibi, H.M.; AlAmiery, A.A.; Gaaz, T.S.: Evaluation and characterization of the symbiotic effect of benzylidene derivative with titanium dioxide nanoparticles on the inhibition of the chemical corrosion of mild steel. Int. J. Corros. Scale Inhib. 2019, 8, 1149

16. Zinad, D.S.; Jawad, Q.A.; Hussain, M.A.M.; Mahal, A.; Shaker, L.M.; Al-Amiery, A.A.: Adsorption, temperature and corrosion inhibition studies of a coumarin derivatives corrosion inhibitor for mild steel in acidic medium: gravimetric and theoretical investigations, Int. J. Corros. Scale Inhib. 2020, 9, 134

17. Al-Amiery, A.; Al-Majedy, Y.K.; Kadhum, A.A.H.; Mohamad, A.B.: New Coumarin Derivative as an Eco-Friendly Inhibitor of Corrosion of Mild Steel in Acid Medium, Molecules 2015, 20, 366

18. Rubaye, A.Y.I.; Abdulwahid, A.A.; Al-Baghdadi, S.B.; AlAmiery, A.; Kadhum, A.A.H.; Mohamad, A.B.: Cheery sticks plant extract as a green corrosion inhibitor complemented with LC-EIS/MS spectroscopy, Int. J. Electrochem Sci. 2015,10, 8200

19. Kadhum, A.; Mohamad, A.; Kadhum, A.A.H.; Mohamad, A.B.; Hammed, L.A.; Al-Amiery, A.; San, N.H.; Musa, A.Y.: Inhibition of Mild Steel Corrosion in Hydrochloric Acid Solution by New Coumarin, Materials 2014, 7, 4335

20. Mohamad, A.; Kadhum, A.; Al-Amiery, A.; Ying, L.; Musa, A.: Synergistic of a coumarin derivative with potassium iodide on the corrosion inhibition of aluminum alloy in $1.0 \mathrm{M} \mathrm{H}_{2} \mathrm{SO}_{4}$, Met. Mater. Int. 2014, 20, 459
21. Zinad, D.S.; Hanoon, M.; Salim, R.D.; Ibrahim, S.I.; AlAmiery, A.; Takriff M.S.; Kadhum, A.A.H.: A new synthesized coumarin-derived Schiff base as a corrosion inhibitor of mild steel surface in $\mathrm{HCl}$ medium: gravimetric and DFT studies. Int. J. Corros. Scale Inhib. 2020, 9, 228

22. Jawad, Q. A.; Zinad, D. S.; Salim, R. D.; Al-Amiery, A.; Gaaz, T. S.; Takriff, M. S.; Kadhum, A.: Synthesis, Characterization, and Corrosion Inhibition Potential of Novel Thiosemicarbazone on Mild Steel in Sulfuric Acid Environment, Coatings 2019, 9, 729

23. Sheet, E.; Yamin, J.; AL-Salihi, H.; Salam, A.; Reda, K. S.; Ahmed, W. K.; Mahdi, M. T.; Al-Amiery, A.A.: N-(3Nitrobenzylidene)-2-Aminobenzothiazole As New Locally Available Corrosion Inhibitor For Iraqi Oil. Industry Journal of the Balkan Tribological Association, 2020, 26, 194

24. Yamin, J.A.A.; Sheet, E.; Al-Amiery, A.: Statistical analysis and optimization of the corrosion inhibition efficiency of a locally made corrosion inhibitor under different operating variables using RSM, Int. J. Corros. Scale Inhib. 2020, 9, 502

25. Jamil, D.M.; Al-Okbi, A.K.; Al-Baghdadi, S.B.; Al-Amiery, A.; Kadhim, A.; Gaaz, T.: Experimental and theoretical studies of Schiff bases as corrosion inhibitors, Chem. Cent. J. 2018, 12, 1

26. Salim, R.D.; Jawad, Q.A.; Ridah, K.S.; Shaker, L.M. AlAmiery, A.; Kadhum, A.A.H.; Takriff, M.S.: Corrosion inhibition of thiadiazole derivative for mild steel in hydrochloric acid solution, Int. J. Corros. Scale Inhib. 2020, 9, 550

27. Al-Amiery, A.; Shaker, L.M.; Kadhum, A.A.H.; Takriff, M.S.: Corrosion Inhibition of Mild Steel in Strong Acid Environment by 4-((5,5-dimethyl-3-oxocyclohex-1-en-1yl)amino)benzenesulfonamide, Tribology in industry $\mathbf{2 0 2 0}$, 42,89

28. Al-Amiery, A.A.; Salman, T.A.; Alazawi, K.F.; Shaker, L.M.; Kadhum A. Takriff, M.S.: Quantum chemical elucidation on corrosion inhibition efficiency of Schiff base: DFT investigations supported by weight loss and SEM techniques. International Journal of Low-Carbon Technologies 2020, 15, 202-209

29. Al-Amiery, A.; Shaker, L. M.; Kadhum, A. A. H.; Takriff, M. S.: Synthesis, characterization and gravimetric studies of novel triazole-based compound. International Journal of Low-Carbon Technologies 2020, 15, 164-170

30. Ogunleye, O.O.; Arinkoola, A.O.; Eletta, O.A.; Agbede, O.O.; Osho, Y.A.; Morakinyo, A.; Hamed, J.O.: Green corrosion inhibition and adsorption characteristics of Luffa cylindrica leaf extract on mild steel in hydrochloric acid environment. Heliyon. 2020, 6, e03205

31. Al-Fakih, A. M.; Abdallah, H.; Aziz, M.: Experimental and theoretical studies of the inhibition performance of two furan derivatives on mild steel corrosion in acidic medium. Materials and Corrosion, 2019, 70, 135-148

32. Jawad Q.A.; Hameed, A.Q.; Abood, M.K.; Al-Amiery, A.A.; Shaker, L.M.; Kadhum, A.A.H.; Takriff, M.S.: Synthesis and comparative study of novel triazole derived as corrosion inhibitor of mild steel in hcl medium complemented with dft calculations. Int. J. Corros. Scale Inhib., 2020, 9, 688-705

33. Chugh, B.; Singh, A. K.; Thakur, S.; Pani, B.; Lgaz, H.; Chung, M.; Jha, R.; Ebenso. E. E.: Comparative Investigation of Corrosion-Mitigating Behavior of Thiadiazole-Deri- 
ved Bis-Schiff Bases for Mild Steel in Acid Medium: Experimental, Theoretical, and Surface Study. ACS Omega, 2020, 5, 13503-13520. doi.org/10.1021/acsomega.9b04274

34. Ji, G.; Shukla, S.; Dwivedi, P.; Sundaram, S.; Ebenso, E.: Green Capsicum Annuum Fruit Extract for Inhibition of Mild Steel Corrosion in Hydrochloric Acid Solution. Int. J. Electrochem. Sci. 2012, 7, 12146-12158

35. Singh, A.; Quraishi, M.: Inhibiting Effects of 5-Substituted Isatin-Based Mannich Bases on the Corrosion of Mild Steel in Hydrochloric Acid Solution. J. Appl. Electrochem. 2010, 40, 1293-1306, DOI: 10.1007/s10800-010-0079-9.

36. Ansari, K.R.; Quraishi, M.; Singh, A.: Isatin Derivatives as a Non-Toxic Corrosion Inhibitor for Mild Steel in 20\% $\mathrm{H}_{2} \mathrm{SO}_{4}$. Corros. Sci. 2015, 95, 62-70

37. Daoud, D.; Douadi, T.; Hamani, H.; Chafaa, S.; Al-Noaimi, M.: Corrosion Inhibition of Mild Steel by Two New S-Heterocyclic Compounds in $1 \mathrm{M} \mathrm{HCl}$ : Experimental and Computational Study. Corros. Sci. 2015, 94, 21- 37, DOI: 10.1016/j.corsci.2015.01.025

38. Kumar, P.; Dahiya, S.; Kumar, R.; Lata, S.; Dahiya, N.; Ahlawat, S.: An exhaustive study of a coupling reagent (1-(3-dimethylaminopropyl) 3-ethylcarbodiimide hydro- chloride) as corrosion inhibitor for steel, Ind. J. Chem. Tech., 2017, 24, 327-335

39. Salarvand, Z.; Amirnasr, M.; Talebian, M.; Raeissi, K.; Meghdadi, S.: Enhanced Corrosion Resistance of Mild Steel in $1 \mathrm{M} \mathrm{HCl} \mathrm{Solution} \mathrm{by} \mathrm{Trace} \mathrm{Amount} \mathrm{of} \mathrm{2-Phenyl-Ben-}$ zothiazole Derivatives: Experimental, Quantum Chemical Calculations and Molecular Dynamics (MD) Simulation Studies. Corros. Sci. 2017, 114, 133-145, DOI: 10.1016/j. corsci.2016.11.002

40. Srivastava, V.; Haque, J.; Verma, C.; Singh, P.; Lgaz, H.; Salghi, R.; Quraishi, M.: Amino Acid Based Imidazolium Zwitterions as Novel and Green Corrosion Inhibitors for Mild Steel: Experimental, DFT and MD Studies. J. Mol. Liq. 2017, 244, 340-352, DOI: 10.1016/j.molliq.2017.08.049

41. Ibrahim, T.; Gomes, E.; Obot, I.; Khamis, M.; Abou Zour, M.: Corrosion inhibition of mild steel by Calotropisprocera leaves extract in a $\mathrm{CO}_{2}$ saturated sodium chloride solution. J. Adhes. Sci. Technol. 2016, 30, 2523-2543, DOI: 10.1080/ 01694243.2016 .1185229

42. Khalil, N.: Quantum chemical approach of corrosion inhibition.Electrochim.Acta.2003,48,2635-2640,DOI: 10.1016/ s0013-4686(03)00307-4 